necessarily indicate that the infant is infected. However, if the ratio of titer of TPPA or RPR for newborn-mother at delivery is more than 4 times, the neonate is considered to be infected with syphilis transmitted from mother. To date, it is not clear for sensitivity and specificity of the quantitative test for TPPA and RPR for diagnosis of congenital syphilis.

Methods Quantitative test for TPPA and RPR in 155 pregnant women with syphilis and their neonates were performed at delivery, and the infants were followed up by 18 months and TPPA was qualitatively tested. The positive of TPPA was considered as diagnosis for congenital syphilis at 18 months after birth. The ratio of titer of RPR or TPPA for newborn-mother was calculated, and their sensitivity and specificity were assessed for diagnosis of congenital syphilis.

Results 27 cases with congenital syphilis were diagnosed. The TPPA titer ratio of neonate-mother was greater than 4 times in 14 cases, of which 13 were patients with congenital syphilis. The sensitivity of TPPA titer ratio of neonate-mother for the diagnosis of congenital syphilis was $48.15 \%(95 \% \mathrm{CI}$ : $29.30 \%-67.00 \%)$. The RPR titer ratio of neonate-mother was greater than 4 times in 7 cases, of which 6 were the patients with congenital syphilis. The sensitivity of RPR titer ratio of neonate-mother for the diagnosis of congenital syphilis was 22.22\%(95\%CI: $6.54 \%-37.90 \%)$. The sensitivity of combination of TPPA and RPR titer ratios for the diagnosis of congenital syphilis was $62.96 \%(95 \% \mathrm{CI}: 44.75 \%-81.18 \%)$. The specificity of the two methods was $99.22 \%$.

Conclusion The sensitivity of RPR or TPPA titer ratio of neonate-mother for the diagnosis of congenital syphilis was low, and the specificity was very high.

Disclosure No significant relationships.

\section{P761 TIP OF THE ICEBERG? AN UNUSUAL SYPHILIS OUTBREAK AMONG YOUNG DUTCH HETEROSEXUALS INDICATING A LARGER EPIDEMIC}

Marita Werner, Anne-Marie Niekamp*, Geneviève Van Liere, Christian Hoebe. Public Health Service South Limburg, Maastricht University Medical Center (MUMC), Sexual Health, Infectious Diseases and Environmental Health, Medical Microbiology, Care and Public Health Research Institute (CAPHRI), Heerlen, Netherlands

\subsection{6/sextrans-2019-sti.819}

Background In 2016, a syphilis outbreak was detected among young heterosexuals in the South of the Netherlands. Syphilis in young Dutch heterosexuals is very rare since many years. Here, we describe the outbreak investigation.

Methods A case was defined as a laboratory confirmed sexually transmitted Treponema pallidum diagnosis between April 2016-December 2017 in individuals reporting heterosexual behavior only, and who were tested in this geographical area. Men having sex with men (MSM), commercial sex workers and swingers were excluded. Outbreak management included raising awareness among GPs and hospitals, extended STI screening, intensified partner notification and enhanced laboratory surveillance. Patients medical records and questionnaires for partner notification were used for analyses.

Results From 2016-2017, 18 cases were identified. Fourteen cases were $<25$ years of whom 9 were $<20$ years, 10 were women. Five cases consulted their GP months prior to diagnosis with symptoms indicating infectious syphilis, but were not diagnosed or adequately treated, causing doctors delay. Two cases were not traceable after diagnosis and not treated. The cases reported 84 sex partners for partner notification, of whom $71 \%$ were aged $<25$. 46\%(39/84) of these sex partners were indeed notified, of whom $30 \%(25 / 84)$ were tested, 5 were diagnosed with syphilis. 54\%(45/84) of the sex partners could not be notified due to lack of contact information. 10 cases could not be linked to other cases and the remaining cases comprised 4 heterosexual couples. No clear source of infection was identified for 14 cases.

Conclusion This unusual outbreak among young heterosexuals indicates a larger epidemic. Continuous transmission is to be expected because most cases could not be linked or their source of infection was not found; only one third of notifiable sex partners were tested despite intensified efforts of partner notification; doctor and patient delay probably caused prolongation of transmission.

Disclosure No significant relationships.

\section{P762 IS KISSING SAFE? DETECTION OF TREPONEMA PALLIDUM IN ORAL SWABS FROM PATIENTS WITH SYPHILIS}

'Lauren Tantalo*, 'Sharon Sahi, ${ }^{2}$ David Katz, ${ }^{3}$ Christina Marra. 'University of Washington, Neurology, Seattle, USA; ${ }^{2}$ University of Washington, Global Health, Seattle, USA; ${ }^{3}$ University of Washington, USA

\subsection{6/sextrans-2019-sti.820}

Background Treponema pallidum (TP) DNA has been detected in oral swabs from patients with early syphilis. The goal of this study was to determine the frequency of detectable TP DNA in oral swabs from patients with all stages of syphilis, and from appropriate controls.

Methods Blood $(\mathrm{N}=131)$, oral swabs $(\mathrm{N}=112)$ and lesion swabs $(\mathrm{N}=72)$ were collected from 138 patients with untreated syphilis (cases). Controls were oral swabs collected from 89 patients presenting to an STD clinic for a concern other than syphilis, $59 \mathrm{HIV}$ positive individuals, and 108 individuals 3,6 or 12 months after treatment of uncomplicated or neurosyphilis (194 samples). DNA was extracted and underwent amplification of a portion of the TP tp0548 gene. Rapid plasma reagin (RPR) tests were performed on serum, and the association between RPR titer and oral TP detection assessed using Mann-Whitney U test.

Results Among those with known syphilis stage, TP was detected in oral swabs from 31/101 (31\%) cases: 4/29 (14\%) with primary, 20/49 (41\%) with secondary, 6/15 (40\%) with early latent, $1 / 7(14 \%)$ with late latent syphilis, and $0 / 1(0 \%)$ syphilis contact. Oral TP was detected in 18/74 (24\%) without detectable blood TP, 6/18 (33\%) without detectable lesion TP, and 21/78 (27\%) without oral lesions. Oral TP detection was more likely with higher RPR titers $(\mathrm{p}=0.002)$. Oral TP was not detected in any of the 342 control samples.

Conclusion Oral TP is detectable in patients at all syphilis stages, most commonly when serum RPR titer is high. Oral TP can be detected even when undetectable in blood or lesion swabs, and in the absence of oral lesions. While detection of DNA is not the same as detection of virulent organisms, these results suggest that individuals with syphilis could infect their partners solely through oral contact.

Disclosure No significant relationships. 